\title{
GAIA Level 1 Maternal Immunization
}

National Cancer Institute

\section{Source}

National Cancer Institute. GAIA Level 1 Maternal Immunization. NCI Thesaurus. Code C127981.

GAIA Level 1 Maternal immunization is defined by three criteria: first, the pregnancy must be confirmed by either a positive pregnancy test or by ultrasound confirmation; second, the date/time of the immunization of the pregnant woman must be recorded in the medical records by the health care worker who administered/witnessed administration of vaccine; third, the details of vaccine must include the lot number and the date of immunization. 